\title{
Circulación y permanencia de las élites parlamentarias desde la perspectiva de género El caso de las cortes valencianas (1983-2011)
}

\author{
Circulation and permanecy of parliamentary elites \\ from a gender perspective \\ The cortes valencianas' case (1983-2011) \\ Bernabé Aldeguer Cerdá • bernabealdeguer@gmail.com \\ UNIVERSIDAD DE ALICANTE
}

Recibido: 16-03-2016

Aceptado: 17-05-2016

\section{Resumen}

La consolidación evidenciada respecto de la incorporación de las mujeres a las instituciones públicas requiere el desarrollo de investigaciones que trasciendan a la mera presencia o representación descriptiva, contribuyendo a dilucidar la eventual incidencia de pautas que, aún en escenarios de presencia equilibrada entre mujeres y hombres, supongan un desigual ejercicio efectivo del cargo. En este sentido, el estudio comparativo de las pautas de circulación y permanencia de las élites parlamentarias desde la perspectiva de género deviene en un cardinal objeto de análisis. El presente trabajo tiene como propósito medir y analizar, de forma comparativa, los factores que inciden en la mayor circulación y menor permanencia en el acta parlamentaria por parte de las mujeres en las Cortes Valencianas durante las siete legislaturas comprendidas entre los años 1983 y 2011. La investigación permite identificar algunos factores explicativos tales como la fragmentación, convergencia y reestructuración partidista en el común espectro ideológico, los resultados electorales (factor oportunidad), el cambio de liderazgo a nivel partidista o parlamentario y los cambios en el rol gobierno u oposición. Se deriva, a la luz de los resultados, la idoneidad de proyectar trabajos similares que permitan ir más allá de la presencia de las mujeres como cargos públicos, mejorando en su caso las metodologías a implementar y, en consecuencia, contribuir a la actualización conceptual sobre el desigual desempeño institucional por razón de género.

Palabras clave: Circulación, Cortes Valencianas, Élites, Género, Paridad, Parlamento, Permanencia

\section{Abstract}

The consolidation concerning to the incorporation of women to the public institutions requires the development of investigations that transcend to the mere presence or descriptive representation, contributing to elucidate the eventual incidence of factors that, still in stages of presence balanced between women and men, suppose an unequal effective exercise of the public office. In this sense, the comparative study on the trends of circulation and permanency of the parliamentary elites from the perspective of gender becomes in a cardinal object of analysis. The aim of the present work consists in the comparative measuring and analysis of the factors that implies a greater circulation and lower permanency in the parliamentary office by women in the Valencian Regional Parliament along the seven legislatures between 1983 and 2011. The research allows to the identification of some explanatory factors such as the organizational fragmentation, convergence and restructuring of political parties in the common ideological spectrum, the electoral results (factor opportunity), the change of leadership into the organizational and parliamentary level, as well as the changes in the government or opposition roles. The study considers the suitability to project similar works that allow to go further of the presence of the women as public offices, sophisticating the methodologies to be implemented and, in consequence, contributing to the conceptual update on the uneven exert institutional by reason of gender.

Key words: Circulation, Elites, Gender, Parity, Parliament, Permanency 


\section{INTRODUCCIÓN}

A la luz de lo aprobado en el marco de la Conferencia Mundial de Mujeres Pekín +10: "se ha prestado mucha atención a la mejora del acceso de la mujer a cargos de decisión política. Sin embargo, se ha prestado mucha menos atención a la permanencia de la mujer en instituciones políticas oficiales (...)” (Declaración Pekín + 10, 2005). Frente a la inquietud y el indudable interés por el estudio descriptivo de la participación política de las mujeres, la atención a las pautas de circulación y permanencia en el cargo deviene en una ineludible tarea dirigida a esclarecer los modelos bajo los cuales hombres y mujeres, de forma diferenciada, consolidan el ejercicio de sus facultades políticas y contribuyen a la adquisición y el ejercicio de la experiencia acumulada.

La Comisión Europea valoró, como uno de los factores significativos para la generación de oportunidades en la incorporación y consolidación de la participación política de las mujeres, la incidencia de las pautas vinculadas a la permanencia y la circulación en el cargo $^{1}$. De acuerdo con el informe Women in European politics -time for action (Comisión Europea, 2009), un elevado índice de permanencia y reelección de los cargos públicos reduce, como consecuencia de la precedente masculinización de la composición descriptiva de las instituciones y el desempeño parlamentario, las oportunidades de incorporación de las mujeres a los parlamentos. Particularmente: "estimates suggest that, on average, around two-thirds of members are re-elected on each occasion meaning that there are limited opportunities for new faces and, therefore, for change in their gender balance" (Comisión Europea, 2009). De esta forma pues, tan relevante resulta el estudio y la promoción de las medidas institucionales que faciliten y permitan la incorporación de las mujeres a los órganos y cuerpos representativos y legislativos por la vía de las correspondientes acciones de discriminación positiva (v. gr., las cuotas de paridad electoral), como la garantía subsiguiente de una continuada y estable permanencia en el cargo de las diputadas, dirigida a acrecentar el nivel de experiencia política paulatinamente atesorado en el ejercicio del ius in officium parlamentario.

Complementariamente al estudio de las élites parlamentarias desde la perspectiva de género por medio de cualquiera de las estrategias de carácter posicional, decisional o reputacional (Putnam, 1976), uno de los factores considerados en el estudio de la participación política de las mujeres en partidos políticos, parlamentos (instituciones públicas representativas),

\footnotetext{
${ }^{1}$ Conviene considerar, tal y como Dahlerup \& Freidenvall ponen de manifiesto, la importancia de las recomendaciones e informes internacionales en materia de paridad y de acceso de las mujeres a los puestos de toma de decisión política. Así: "the discoursive framing has here centred around democratisation and adjustment to international norms on representation” (Dahlerup \& Freidenvall, 2008). En particular, Connell destaca la importancia de las Conferencias de la Organización de Naciones Unidas (ONU) durante las últimas tres décadas a la hora de comprender cómo se han transformado las agendas regionales y nacionales, hacia un mayor impacto sobre las cuestiones de género (Connell, 2002). De forma esencial, un apartado como el presente resulta inevitable en la medida en que siguiendo a Sevilla Merino: "los conceptos de discriminación y de acción positiva provienen del derecho internacional, que las define, en uno de los primeros textos que las regulan, como "medidas especiales destinadas a tener en cuenta la situación particular de personas para las que se considera generalmente necesaria una protección o una asistencia especial por razones como el sexo, la edad, la invalidez, las cargas familiares o el nivel social y cultural” (Convenio núm. 111 Organización Internacional del Trabajo, 1958, referente a la discriminación en el empleo)” (Sevilla Merino, 2004).
} 
gobiernos y Administraciones Públicas, ha venido dado pues, por las pautas de circulación y permanencia temporal en el cargo, bien sea con propósitos exclusivos o complementarios de carácter descriptivo, explicativo, predictivo o evaluativo. La circulación de las elites constituye un componente central en el desarrollo de la propia teoría de las élites, sirviendo de referencia al respecto la elocuente expresión de Pareto $(1932,1980)$ al definir la Historia como un cementerio de aristocracies, cuya renovación (y pautas de permanencia también) permite interpretar el transcurso de los acontecimientos políticos con carácter diacrónico.

De esta forma, han resultado de especial interés las relaciones entre las pautas de circulación y permanencia, el tamaño de los distritos electorales o la institucionalización del proceso de reclutamiento y selección en el seno de los partidos políticos, en tanto que factores útiles y significativos a la hora de analizar las oportunidades y los obstáculos institucionales que operan en el acceso de las mujeres a los parlamentos (Githens, 2003). Githens respalda la puesta en marcha de estudios e investigaciones que testen la relevancia de la variable género en los diferentes momentos y estadios del proceso de reclutamiento y selección, lo cual remite igualmente a la incidencia sobre las pautas de circulación y permanencia. En este sentido, los factores institucionales o de la demanda resultan fundamentales a la hora de valorar el papel de los partidos políticos como agentes o Stakeholders en la configuración de las instituciones públicas².

Las distintas estrategias metodológicas dirigidas a generar, interpretar y evaluar los datos asociados a la circulación y la permanencia de los cargos públicos, permiten extraer las claves fundamentales que desde un punto de vista descriptivo y explicativo permiten avanzar en la noción relativa al ejercicio pleno y efectivo que del cargo realizan las mujeres en tanto que diputadas con respecto de sus homólogos varones. Se pretende trascender así la propia representación descriptiva en términos de Pitkin irrumpiendo en la propia dimensión substantiva del discurso político a favor de la igualdad de oportunidades de mujeres y hombres (Pitkin, 1985; Sapiro, 1998; Paxton \& Hughes, 2007).

Más allá de la propia presencia que de forma gradual se dé en relación a la presencia de las mujeres en el seno de las instituciones parlamentarias, resulta preciso ahondar en el detalle de las pautas que caracterizan el ejercicio real de las facultades ligadas a la titularidad del acta parlamentaria, siendo la circulación y la permanencia en el cargo una de las estrategias útiles a tal efecto. En última instancia, es de esta forma como resulta posible ahondar en esos: “(...) fenómenos que dificultan un equilibrio de género real y efectivo en el ejercicio del poder político, tales como la renovación más alta de las mujeres que de hombres (...)” (Verge, 2006).

\footnotetext{
${ }^{2}$ Además, tal y como destaca en su trabajo Rodríguez Teruel al escrutar, de forma específica, la circulación de las élites en el seno de los gabinetes gubernamentales, "el análisis del personal político que opera en una determinada institución aporta una información valiosa sobre el funcionamiento de esa institución (canales de acceso y reclutamiento, factores de restricción, circulación interna, grado de institucionalización...) y con ese objetivo se han dirigido la atención hacia el parlamento, los altos cargos de las administraciones públicas o los partidos” (Rodríguez Teruel, 2011).
} 
Especialmente, dicha permanencia y más estable y continuada representación de las mujeres en las instituciones públicas, en tanto que un parámetro o indicador de la mayor democratización del sistema político (Mansbridge, 1998; Phillips, 1998; Lovenduski, 2005; Squires, 2007), no supondría sin embargo controvertir una elevada tasa de circulación en tanto que "signo de buen funcionamiento del sistema democrática que impediría la perpetuación de los líderes en el poder” (Uriarte, 1997). Más bien al contrario, la adquisición de experiencia por parte de las mujeres devendría en un acrecentamiento de las oportunidades por medio de la consolidación de sus discursos a favor de la igualdad de género, así como la más equilibrada y justa representación según sexos a la luz del concepto de democracia paritaria (Declaración de Atenas, 1992; Sevilla Merino, 2004; Pastor, 2011). El sexo, junto al lugar de nacimiento (urbano vs. rural), la clase social o el nivel de formación, constituyen variables que inciden en el reclutamiento y el acceso a las élites, de tal forma que ser hombre deviene en un rasgo que aumenta "las posibilidades de poder alcanzar posiciones de élite política” (Uriarte, 1997).

A la luz de lo antedicho, la deseable mayor permanencia de las diputadas en el cargo, en orden a promover la estabilidad de una creciente e ininterrumpida continuidad en el ejercicio de las facultades parlamentarias, deviene en una premisa clave para asegurar:

- Una gradual proyección y presencia mediática como resultado de la posición política ejercida a nivel institucional como consecuencia de ostentar la titularidad de un acta parlamentaria;

- Una mayor profesionalidad, conocimiento y dominio de las prácticas políticas asociadas al ejercicio efectivo del cargo, tanto a nivel parlamentario como gubernamental;

- Una ampliación y consolidación de las redes de acción y colaboración en función del grado de penetración en la estructura partidista y del grupo político institucional (grupo parlamentario o consejería autonómica) a nivel social y político; y,

- Como consecuencia y resultado acumulativo de las citadas tres dimensiones previas, cabe considerar el incremento en las posibilidades de, no solo acceder al cargo, sino de ejercer el liderazgo político que del mismo se pueda derivar sucesivamente, perpetuando de este modo las posibilidades de la reelección y la continuidad conforme a desempeño acreditado y reconocido a nivel social, mediático, orgánico-partidista e institucional (representativo o gubernamental) ${ }^{3}$.

La reelección de los diputados (hombres) y su consiguiente mayor permanencia en el cargo deviene, como causa por una parte, y consecuencia o efecto además, en una tendencia ligada a generar y favorecer una mayor exposición mediática de los mismos. La estrategia de la coalición o partido político fundamentada en las pretensiones de incrementar las pro-

\footnotetext{
${ }^{3}$ Si bien, conforme a Morán: "la ausencia de renovación elimina el grado de permeabilidad necesario entre Parlamento, sistema político y sistema social, y afecta también de un modo negativo al proceso de institucionalización” (Morán, 1989).
} 
babilidades de obtener un mejor resultado electoral conlleva la selección de aquellos/as candidatos/as con mayor experiencia acumulada y/o proyección mediática. En virtud de tal presunción estratégica, se reducen las posibilidades de que se produzca, ya no sólo la irrupción de nuevas diputadas en el ámbito institucional y mediático en una secuencia de consolidación tras una primera fase de incorporación, sino la propia permanencia o continuidad en un lapso sucesivo de estabilidad en el cual quedan favorecidas las opciones logradas por los diputados, ostentando reiteradamente de las posiciones clave de las candidaturas electorales (cabezas de lista, primeros tramos y safe sites).

El presente trabajo tiene como propósito el estudio comparativo de las pautas de circulación y permanencia de los diputados y de las diputadas autonómicas de las Cortes Valencianas con carácter diacrónico a la luz de factores tales como los cambios de liderazgo en el seno del correspondiente partido político o el rol ejercido por cada grupo parlamentario desde la perspectiva de las tareas ligadas al gobierno o a la oposición. La hipótesis de trabajo se formula en el sentido de pronosticar una mayor tasa de circulación entre las diputadas con respecto a los diputados, en la medida en que la más elevada y consolidada profesionalización de los diputados por la vía de la permanencia en el cargo, conculca las posibilidades y las opciones de nominación, acceso y consolidación de las mujeres en tanto que candidatas y, consecuentemente, como diputadas.

Los factores que se considerarán en el estudio y la evaluación longitudinal de las pautas de renovación y permanencia de las diputadas autonómicas valencianas serán los siguientes:

- Los cambios de liderazgo en el seno de la organización partidista (coalición o partido político).

- La evolución de los resultados electorales como parámetro para evaluar la mayor o menor representación parlamentaria, y de esta forma, la incidencia en la propia composición de los distintos grupos parlamentarios (tanto de mujeres como de hombres) en términos numéricos y de oportunidades para el acceso a las labores de gobierno, deviniendo eventuales cambios de mayorías y, en consecuencia, los correspondientes traspasos de poder y los cambios de la oposición al gobierno, o viceversa. En este sentido, conforme a Putnam, la circulación o renovación se relaciona de forma directamente proporcional con los contextos de crisis e inversamente con los períodos de estabilidad (Putnam, 1976).

- La propia incorporación disruptiva o el umbral de presencia de mujeres en los diferentes grupos parlamentarios y en el conjunto de la cámara parlamentaria de forma agregada.

La cuestión clave sobre la que la investigación se propone dilucidar para el caso valenciano reside en esclarecer la existencia y el grado en que opera un eventual nivel de superior permanencia en el cargo entre los diputados en comparación con las diputadas. Dada la importancia de la variable asociada a la circulación y permanencia de las élites políticas desde una perspectiva comparada por sexos, se propone en el presente trabajo la medición del índice de circulación de los/as diputados/as autonómicos/as valencianos/as. 
La circulación ha sido mensurada teniendo en consideración la propuesta metodológica utilizada en otras investigaciones (Coller, 1999; Verge, 2006), en virtud de las cuales, siguiendo a Coller (1999): "la tasa de circulación de la legislatura se ha calculado dividiendo el número de parlamentarios que no provienen de la legislatura precedente por el total de diputados de esa legislatura” (Coller, 1999). Se considera la circulación únicamente en relación al acta parlamentaria, no valorándose la continuidad en otras arenas o espacios de representación y de gobierno, o en su caso, el abandono definitivo que de toda responsabilidad institucional pudiera darse en términos de Blondel, Müller-Rommel \& Malová (2007).

\section{UNA APROXIMACIÓN PRELIMINAR A LA INCORPORACIÓN DE LAS MUJERES A LAS CORTES VALENCIANAS}

Con objeto de contextualizar los análisis sobre la circulación y permanencia de las diputadas autonómicas valencianas, se expone en el presente epígrafe el estudio de la presencia de las mismas en el cómputo global de las candidaturas, el acceso efectivo al cargo (en tanto que diferencia entre el porcentaje de candidatas y el de diputadas que acceden al cargo) y la definitiva presencia en la institución parlamentaria autonómica (Cortes Valencianas).

La superación del umbral de masa crítica ${ }^{4}$ acontecerá, para el conjunto de la Comunidad Valenciana, de la mano de Esquerra Unida del País Valencia (en adelante, EUPV) en la III (1991) y IV (1995) Legislaturas con un 33.73 \% y un $39.15 \%$ de mujeres, respectivamente. El resto de formaciones (Partido Socialista del País Valencià -en adelante, PSPV- y Partido Popular de la Comunidad Valenciana -en adelante, PPCV-) pasarán de una legislatura a otra desde umbrales por debajo del $30.00 \%$ hasta los propios del umbral de presencia equilibrada (40.00 \%) de manera directa. Así, dicha transición será experimentada por el PSPV entre la III (29.27 \%) y la IV Legislaturas (40.74 \%), y por el PPCV entre la IV (27.98 \%) y la V Legislaturas (45.25\%). Cabe destacar que, una vez superado el umbral de presencia equilibrada (40.00 \%) por parte de todas las candidaturas en el conjunto de la Comunidad Valenciana, ninguna formación ha evidenciado un descenso en la presencia las mujeres por debajo del $40.00 \%$. Así, la tendencia relativa al incremento global de las mujeres en las candidaturas ha venido dada por una primera fase de aumento destacado que da paso a la superación de los umbrales de masa crítica y de equilibrio, y que sucede en el PSPV de la III a la IV Legislatura, y en el PPCV y EUPV de la IV a la V Legislatura.

Una vez en el umbral de la presencia equilibrada, la tendencia ha sido monocromática con carácter ascendente y por tanto ininterrumpida en el caso de EUPV, aunque no así para el

\footnotetext{
${ }^{4}$ El concepto de masa crítica, de acuerdo con Paxton \& Hughes: “(...) suggest that when women reach a certain percentatge of a legislatura, they will be better able to pursue their policy priorities and legislative styles. (...) If women reach a critical mass and more out of taken status, the increased influence of women and a feminization of the political agenda will be seen” (Paxton \& Hughes, 2007). De acuerdo con Lois González, la idea de masa crítica permite identificar un umbral de presencia las mujeres a partir del cual se observan e introducen cambios en dimensiones tales como el discurso político, la agenda y la cultura política (Lois González, 2007). Así, desde una perspectiva proactiva y normativa: “(...) és necessari que existeixi un mínim, una massa crítica de dones per tal de generar resistència davant la pressió per socialitzar-se en els comportaments masculins establerts” (Verge, 2009).
} 
PSPV y el PPCV, con un descenso entre la V y la VI Legislatura (más acusado en el PSPV que en el PPCV, aun cuando la presencia las mujeres será superior en el PSPV no obstante), y un nuevo incremento entre la VI y la VII Legislatura (más destacado en el PSPV que en el PPCV).

Tabla 1. La presencia de las mujeres (N - \%) en el cómputo global de las listas electorales autonómicas por partidos políticos y legislaturas (1991 - 2007) (Provincia de Alicante)

\begin{tabular}{cccccc} 
& III Leg. & IV Leg. & V Leg. & VI Leg. & VII Leg \\
& $91 / 95$ & $95 / 99$ & $99 / 03$ & $03 / 07$ & $07 / 11$ \\
PSPV & 9 & 15 & 14 & 18 & 20 \\
\hline & $26,47 \%$ & $44,12 \%$ & $41,18 \%$ & $52,94 \%$ & $50,00 \%$ \\
\hline PPCV & 8 & 15 & 15 & 19 & 17 \\
\hline & $23,53 \%$ & $44,12 \%$ & $44,12 \%$ & $55,88 \%$ & $42,50 \%$ \\
\hline & 13 & 15 & 16 & 14 & 18 \\
\hline
\end{tabular}

Fuente: Elaboración propia a partir de Acuerdos de las Juntas Electorales Provinciales de Alicante, Castellón y Valencia sobre proclamación de las candidaturas concurrentes a las Elecciones a las Cortes Valencianas publicadas en los correspondientes Boletines Oficiales de la Provincia.

Gráfico 1. La presencia de las mujeres (N - \%) en el cómputo global de las listas electorales autonómicas por partidos políticos y legislaturas (1991 - 2007) (Provincia de Alicante)

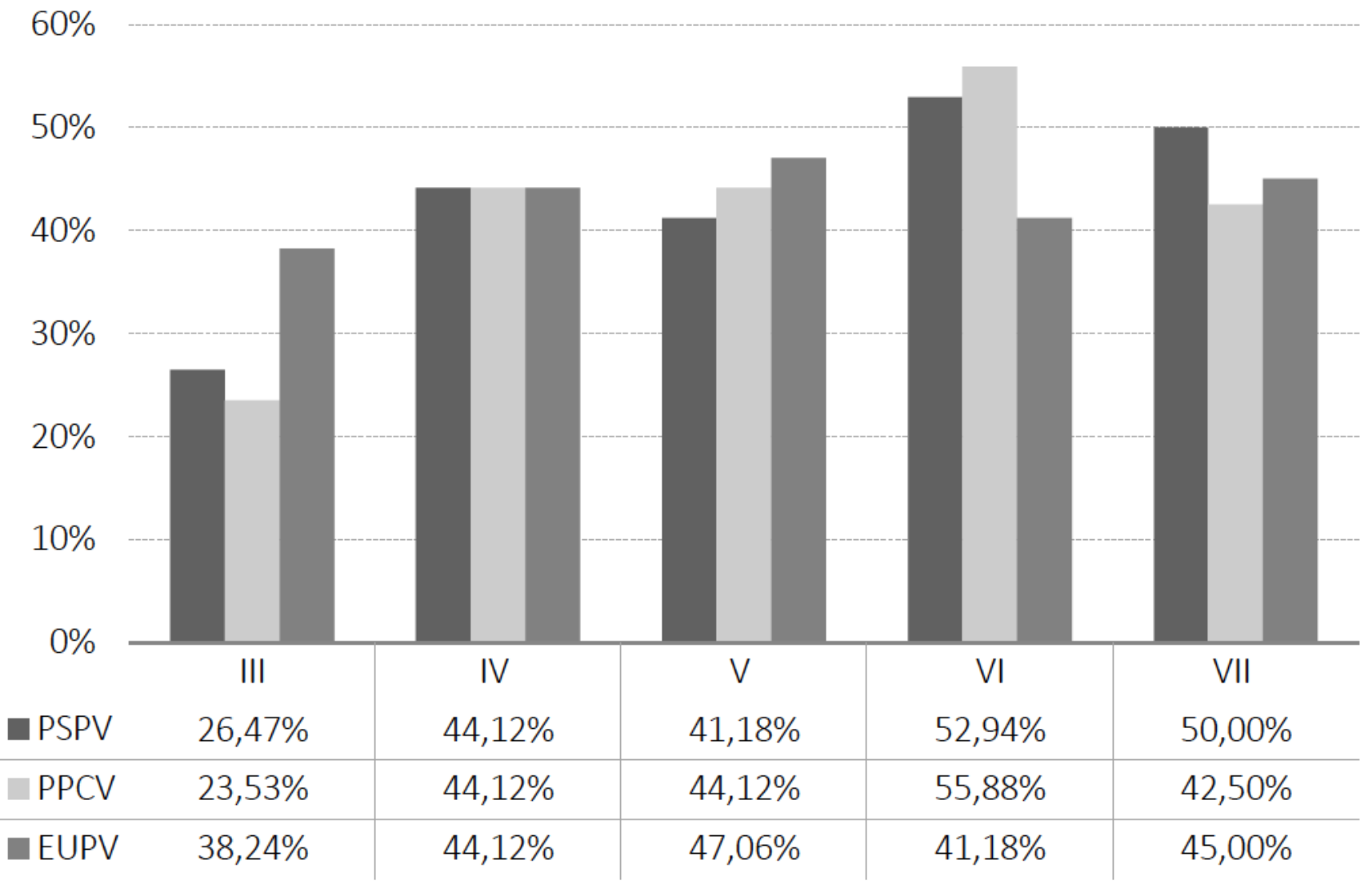

Fuente: Elaboración propia a partir de Acuerdos de las Juntas Electorales Provinciales de Alicante, Castellón y Valencia sobre proclamación de las candidaturas concurrentes a las Elecciones a las Cortes Valencianas publicadas en los correspondientes Boletines Oficiales de la Provincia. 
El acceso efectivo de las mujeres a las Cortes Valencianas en el seno del PSPV muestra una clara tendencia ascendente a lo largo de la secuencia histórica con carácter monocromático, de forma particular, a partir de la V Legislatura, siendo en esta convocatoria la tasa negativa más reducida en cuanto a los registros de acceso efectivo (-4.2 en la V Legislatura), siendo a partir de dicha legislatura cuando se experimente una subida paulatina que ubicará el indicador de acceso efectivo en registros positivos; a saber, siendo superior el porcentaje de diputadas electas que el de candidatas en el conjunto de las candidaturas del PSPV para las tres provincias (Alicante, Castellón y Valencia). Será precisamente en esta V Legislatura cuando, respecto de la anterior (IV), se experimente el mayor incremento en cuanto al acceso efectivo, aun sin superar el umbral en el marco de los registros positivos. Ello se produce en coincidencia con la consolidación de la presencia las mujeres, tanto en el conjunto de las Cortes Valencianas como, en particular, en los grupos parlamentarios del PSPV, tras la superación del umbral de presencia equilibrada o de paridad (40.00 \%). En la VI Legislatura, el índice de acceso efectivo pasará a ser positivo, en coincidencia con una presencia de las mujeres del $45.71 \%$ en los puestos seguros. Si bien, tras la V Legislatura, en la VI y VII se experimentará una estabilización en el indicador de acceso efectivo en el umbral positivo, no superando sin embargo, el 0.05 en la VI y el 0.1 en la VII.

Por otro lado, en el PPCV se evidencia una subida creciente y consolidada en cuanto al acceso efectivo, si bien, siempre en índices negativos que nunca se ubicarán por encima del umbral del 0.00, no siendo en ninguna legislatura superior el porcentaje de diputadas que el de candidatas para las sucesivas convocatorias electorales. No obstante ello, la tendencia paulatina de crecimiento resulta reducida pero constante y experimenta su mayor subida en la VI Legislatura, a pesar de ser ésta en la que se registra un descenso en el porcentaje de mujeres presentes en su grupo parlamentario. Con todo, en la última legislatura de la secuencia longitudinal considerada la tendencia cambia hacia una consolidación de los registros negativos en el indicador de acceso efectivo hasta el -4.26.

Finalmente, en EUPV se destaca una variación y un comportamiento inestable de los datos que lleva a descensos constantes en el índice de acceso efectivo desde la IV hasta la VI y un ascenso significativo en la VII. De nuevo, se pone de manifiesto el impacto de los resultados electorales obtenidos (factor de oportunidad) sobre la posibilidad a favor (o en contra) de las mujeres a la hora de acceder a las instituciones, afectando así y en consecuencia al índice de acceso efectivo.

En suma, el sistema de cuotas obligatorias a partir del año 2007 ha derivado en unos más elevados índices de acceso efectivo para el conjunto de la secuencia histórica, tanto para el PSPV como para EUPV, y un ligero descenso en el ya de por sí crónico registro negativo en el índice de acceso efectivo para las candidatas del PPCV. En cualquier caso, la desigual ubicación de hombres y mujeres en los puestos seguros o puestos elegibles afecta, tal y como Ortega, Torres y Trujillo evidencian para el caso nacional (Congreso de los Diputados), a los índices de renovación y permanencia, en la medida en que se otorga preferencia a los hombres sobre las mujeres en las listas electorales, tanto en los puestos de renovación como en los que implican un incremento de la representación (Ortega, Torres y Trujillo, 2011). 
Tabla 2. El indicador de Acceso efectivo a las Cortes Valencianas por parte de las diputadas autonómicas valencianas, por partidos políticos y legislaturas (Comunidad Valenciana) (\%)

\begin{tabular}{lccccc} 
& III Leg. & IV Leg. & V Leg. & VI Leg. & VII Leg \\
& $91 / 95$ & $95 / 99$ & $99 / 03$ & $03 / 07$ & $07 / 11$ \\
\hline PSPV & $-10,64$ & $-12,96$ & $-4,2$ & $+0,05$ & $+0,10$ \\
\hline PPCV & $-10,35$ & $-9,94$ & $-8,54$ & $-2,96$ & $-4,26$ \\
\hline EUPV & $-22,62$ & $+1,96$ & $-9,98$ & $-32,73$ & $+9,14$
\end{tabular}

Fuente: Elaboración propia a partir de Acuerdos de las Juntas Electorales Provinciales de Alicante, Castellón y Valencia sobre proclamación de las candidaturas concurrentes a las Elecciones a las Cortes Valencianas publicadas en los correspondientes Boletines Oficiales de la Provincia y acuerdos de la Junta Electoral de la Comunidad Valenciana, por las que se publican los resultados finales y la relación de diputados y diputadas electos en las elecciones a las Cortes Valencianas.

Gráfico 2. El indicador de Acceso efectivo a las Cortes Valencianas por parte de las diputadas autonómicas valencianas, por partidos políticos y legislaturas (Comunidad Valenciana) (\%)

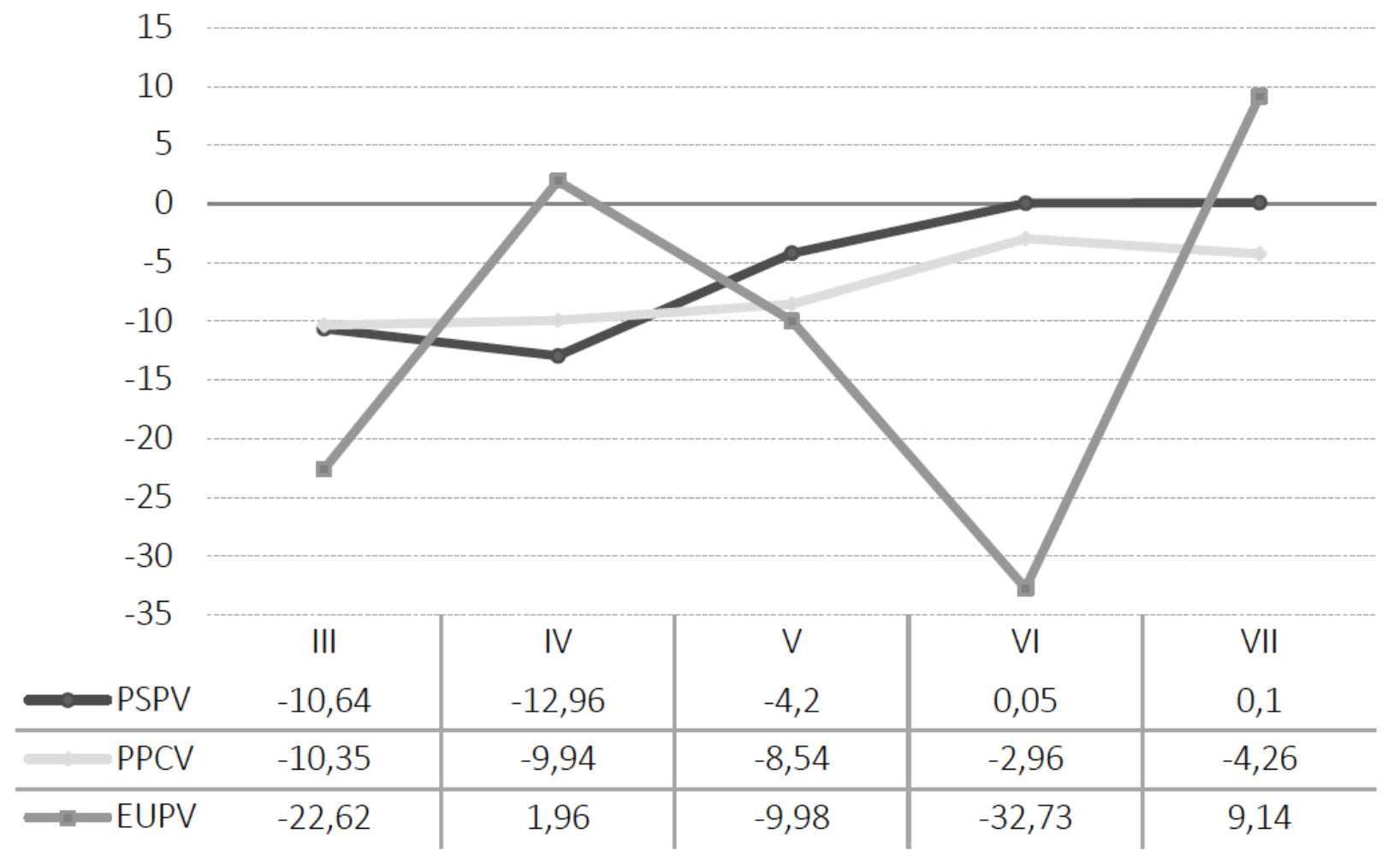

Fuente: Elaboración propia. Acuerdos de las Juntas Electorales Provinciales.

Para el conjunto de la cámara parlamentaria, se observa que, en el cómputo agregado de toda la secuencia histórica (1983 - 2011), la representación las mujeres se ha ubicado en niveles inferiores a los propios del umbral de masa crítica y, por tanto, muy por debajo de la horquilla de la presencia equilibrada del 40 - 60 \%. Las mujeres han constituido así un $25.63 \%$ del conjunto de los/as parlamentarios/as a lo largo de la perspectiva cross-time que se comprende en la investigación. Si bien, dicha presencia ha experimentado cuatro fases con carácter evolutivo: 
- Primer período. Presencia testimonial o fase de exclusión, con cinco mujeres en las dos primeras legislaturas, constituyendo para ambos períodos (1983/1987 y 1987/1991), el $5.62 \%$ de los miembros de las Cortes Valencianas.

- Segundo período. Abarca el período comprendido entre la III (1991/1995) y IV legislaturas (1995/1999), con una presencia que, sin superar el umbral de masa crítica (30.00\%), sí implica no obstante, una ruptura de la pauta de exclusión y presencia testimonial propia de las dos primeras legislaturas (de 1983 a 1991). Es de forma particular a partir de la III Legislatura el instante en que, para el análisis de las candidaturas electorales, es posible la obtención de conclusiones representativas desde un punto de vista comparativo entre sexos, pues hasta tal momento, el gap de los datos en relación a tal variable imposibilita cualquier análisis comparativo. Así, en la III Legislatura (1991/1995) se supera, doblándose, la presencia de las mujeres registrada en la legislatura inmediatamente previa, lo cual supondrá alcanzar el 13.48 \% de diputadas sobre el total, porcentaje que se incrementará hasta el 24.72 \% en la IV Legislatura (1995/1999) con 22 mujeres sobre un total de 89 parlamentarios/as autonómicos. Ello es coherente con el incremento destacado que comienza a darse en la III y la IV Legislaturas en las candidaturas electorales.

- Tercer período. En la V y VI Legislaturas se produce una presencia de las mujeres que es, respectivamente, del 40,45 y del 42,70 \%, lo cual supone una superación del umbral, no sólo de masa crítica (del $30.00 \%$ ), sino del considerado como constitutivo de una presencia equilibrada entre mujeres y hombres (40.00 \%).

- Cuarto período. Finalmente, en la VII Legislatura (2007/2011), tras la aplicación de las disposiciones electorales de paridad (cuotas) conforme a la Disposición Adicional Segunda de la Ley Orgánica de Igualdad Efectiva entre Mujeres y Hombres (en adelante, LOIEMH), se va a producir una presencia de las mujeres que supera la tasa del $49 \%$ (49.44 \%) y que, por tanto, lleva a una situación de paridad en el seno de las Cortes Valencianas.

Según los datos y su comportamiento en la secuencia cross time, la presencia de las mujeres en las Cortes Valencianas ha implicado un incremento continuado e ininterrumpido de acuerdo con las cuatro fases más arriba detalladas, marcadas por dos hitos significativos:

- En primer lugar, la adopción de las cuotas voluntarias o cuotas blandas por parte del PSPV (1997) y de EUPV (1999), y que con vigencia de las mismas en la convocatoria electoral de la V Legislatura (1999/2003), y el efectivo imitación o contagio por parte del PPCV5, va a suponer la superación, no sólo del umbral de masa crítica, por encima del 30.00 \% para el conjunto del Pleno y de cada uno de los grupos parlamentarios, sino incluso llegando a la satisfacción del principio de presencia equilibrada para el total del Pleno (con un 40.45 $\%$ de mujeres) y por parte del PSPV (42.86 \%) y EUPV (40.00 \%). El PPCV dispondrá de un

\footnotetext{
${ }^{5}$ Siguiendo a Sevilla Merino: “en el contexto mundial, el estudio llevado a cabo por la Unión Interparlamentaria estima: 1. Que se produce un fenómeno de arrastre que hace que se generalice la tendencia a la feminización de los partidos políticos, sobre todo en el caso de que la competencia electoral entre los mismos sea fuerte; y, 2. Que los partidos que se autodenominan de izquierdas y los alternativos tienen mayor propensión a presentar y elegir más mujeres que los restantes” (Sevilla Merino, 1997).
} 
$38.76 \%$ de mujeres en el seno de su grupo parlamentario para la V Legislatura, lo cual implica un acercamiento evidente al umbral de representación equilibrada.

- En segundo lugar, la aplicación de las disposiciones electorales de cuotas legislativas, legales u obligatorias, en la convocatoria electoral dela VII Legislatura (2007), que implicará una presencia las mujeres en el conjunto de las Cortes Valencianas próxima al $50.00 \%$ (en concreto, del $49.99 \%$ ), nivel porcentual al que contribuirán, fundamentalmente, el $50.00 \%$ de diputadas en el grupo parlamentario del PSPV, el $57.14 \%$ en el de EUPV y, el más limitado $38.89 \%$ en el del PPCV. Se observa, pues, una correlación positiva y claramente significativa entre la puesta en marcha de instrumentos institucionales, tanto en el seno de los partidos políticos como en el entramado jurídico de carácter electoral, y el comportamiento de los actores políticos en cuanto a la incorporación de las mujeres a las instituciones públicas representativas (Cortes Valencianas, en nuestro caso).

Tabla 3. Los períodos en la incorporación de las mujeres a las Cortes Valencianas $(1983-2011)$

\begin{tabular}{ll} 
Períodos & Presencia de las mujeres (\%) \\
\hline Primer período: Exclusión / presencia testimonial & I Legislatura: $5,62 \%$ \\
Segundo período: Superación de la exclusión / presencia testimonial & II Legislatura: $5,62 \%$ \\
\hline $\begin{array}{l}\text { Tercer período: Masa crítica y representación equilibrada } \\
\text { (Cuotas voluntarias en PSPV y EUPV) }\end{array}$ & IV Legislatura: $24,72 \%$ \\
\hline $\begin{array}{l}\text { Cuarto período: paridad. } \\
\text { (Cuotas legales: LOIEMH) }\end{array}$ & VI Legislatura: $40,45 \%$ \\
\hline
\end{tabular}

Fuente: Elaboración propia a partir de Archivo de las Cortes Valencianas.

Tabla 4. La composición del Pleno de las Cortes Valencianas por sexos (1983 - 2011)

\begin{tabular}{|c|c|c|c|}
\hline & \multicolumn{2}{|c|}{ Sexo } \\
\hline & & $M$ & $\mathrm{H}$ \\
\hline \multirow{2}{*}{ I Legislatura } & $\mathrm{N}$ & 5 & 87 \\
\hline & $\%$ & 5,62 & 94,38 \\
\hline \multirow{2}{*}{ II Legislatura } & $\mathrm{N}$ & 5 & 87 \\
\hline & $\%$ & 5,62 & 94,38 \\
\hline \multirow{2}{*}{ III Legislatura } & $\mathrm{N}$ & 12 & 77 \\
\hline & $\%$ & 13,48 & 86,52 \\
\hline \multirow{2}{*}{ IV Legislatura } & $\mathrm{N}$ & 22 & 67 \\
\hline & $\%$ & 24,72 & 75,28 \\
\hline \multirow{2}{*}{ V Legislatura } & $\mathrm{N}$ & 36 & 53 \\
\hline & $\%$ & 40,45 & 59,55 \\
\hline \multirow{2}{*}{ VI Legislatura } & $\mathrm{N}$ & 38 & 50 \\
\hline & $\%$ & 42,70 & 57,30 \\
\hline \multirow{2}{*}{ VII Legislatura } & $\mathrm{N}$ & 44 & 55 \\
\hline & $\%$ & 49,44 & 50,56 \\
\hline \multirow{2}{*}{ Total Secuencia Histórica (1983 - 2011) } & $\mathrm{N}$ & 162 & 470 \\
\hline & $\%$ & 25,63 & 74,37 \\
\hline
\end{tabular}

Fuente: Elaboración propia a partir de Archivo de las Cortes Valencianas. 
Gráfico 3. La composición del Pleno de las Cortes Valencianas por sexos (1983 - 2011) (\%)

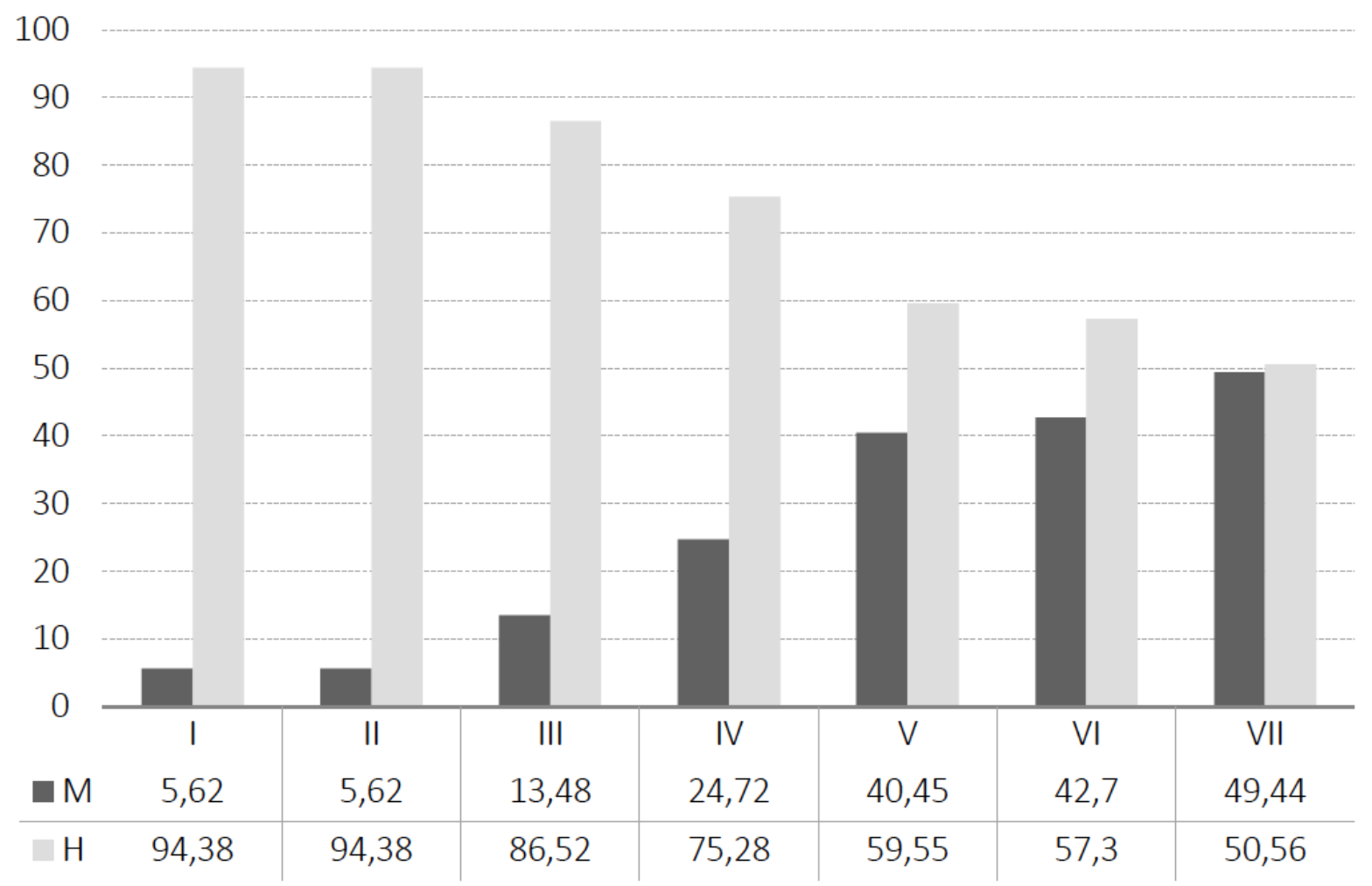

Fuente: Elaboración propia a partir de Archivo de las Cortes Valencianas.

\section{EL ESTUDIO COMPARATIVO CROSS-TIME (1983-2011) POR SEXOS Y GRUPOS PARLAMENTARIOS DE LAS PAUTAS DE CIR- CULACIÓN Y PERMANENCIA DE LAS ÉLITES PARLAMENTA- RIAS DE LAS CORTES VALENCIANAS}

\subsection{Las pautas de circulación por sexos y grupos parlamentarios (1983-2011)}

De acuerdo con los resultados obtenidos al medir la tasa de circulación de las élites parlamentarias de las Cortes Valencianas por sexos para el conjunto de los grupos parlamentarios en cada una de las legislaturas, cabe constatar una mayor tasa de circulación entre las diputadas (0.64) de forma comparativa con respecto a los diputados (0.55).

Es preciso señalar, en cualquier caso, que la única excepción que se produce en dicha tendencia diacrónica acaecerá en la II Legislatura (1987-1991), en la que el índice de circulación de las mujeres manifiesta una cifra excepcionalmente baja en relación al resto de legislaturas. Ello se debe a la permanencia en el testimonial grupo de diputadas presentes en la cámara para el conjunto de las dos primeras y sucesivas legislaturas (1983-1987 y 1987-1991).

La diferencia entre los índices de circulación de diputados y diputadas para cada una de las sucesivas legislaturas consideradas permite apreciar una reducción paulatina de tal indicador en la medida en que se avanza en la secuencia longitudinal, especialmente a partir de la IV Legislatura (1995-1999). De este modo, es posible considerar dos momentos diferencia- 
dos a lo largo de la secuencia longitudinal en virtud del punto de inflexión que constituye el citado período 1995-1999, en el cual el índice de circulación de las diputadas mostrará un pico histórico con respecto a otros períodos, con la mayor diferencia registrada comparativamente en relación a los diputados. A continuación se enumeran los tres factores que permiten comprender los datos antedichos relativos a la IV Legislatura (1995-1999); a saber:

- Se trata del períodos de incorporación o incremento en la presencia de las mujeres en el conjunto de las Cortes Valencianas;

- Se dará lugar a diferentes cambios de liderazgo en el seno delos diferentes partidos políticos y coaliciones, y consecuentemente de los correspondientes grupos parlamentarios; y,

- Acontecerán cambios de mayorías políticas parlamentarias y, por consiguiente, alternancia de gobierno.

A pesar de la diferencia en el índice de circulación en el sentido señalado (mayor entre las diputadas que entre los diputados), el comportamiento de los datos es correlativo para ambos sexos desde una perspectiva longitudinal, excepto en aquellos lapsos de la secuencia histórica en que, como en la IV Legislatura (1995-1999), se registra una mayor tasa de circulación entre las mujeres, debido a que es en este período cuando se dará, por primera vez, el acceso de las mujeres a las Cortes Valencianas, pasando de la presencia testimonial del 5.62 \% en la II (1987-1991) y del 13.48 \% en la III (1991-1995), al 24.72 \% en la IV legislatura (19951999), en coincidencia con la adopción de cuotas voluntarias en el seno del PSPV-PSOE y de EUPV-IU.

Tabla 5. Media ponderada de la tasa de circulación de las élites parlamentarias de las Cortes Valencianas, por sexos y legislaturas, para el conjunto de los grupos parlamentarios

\begin{tabular}{lccc} 
Legislatura & M & H & Diferencia \\
II & 0,33 & 0,48 & $-0,15$ \\
\hline III & 0,75 & 0,59 & 0,16 \\
\hline IV & 0,82 & 0,56 & 0,26 \\
\hline V & 0,69 & 0,61 & 0,08 \\
\hline VI & 0,47 & 0,48 & 0,01 \\
\hline VII & 0,54 & 0,57 & 0,03 \\
\hline TOTAL & 0,64 & 0,55 & 0,09 \\
\hline
\end{tabular}

Fuente: Elaboración propia a partir de Archivo de las Cortes Valencianas.

La realización del análisis por grupos parlamentarios muestra unas pautas coherentes con los resultados obtenidos y sistematizados en relación al conjunto de la cámara. Si bien, a continuación se abordan las particularidades y especificidades que permiten interpretar la diferente evolución del índice de circulación en cada uno de los diferentes grupos parlamentarios a la luz de las tres variables señaladas más arriba; a saber: incorporación e incremento de presencia de las mujeres; cambios de liderazgo; y, cambios de gobierno y/o de mayorías parlamentarias. 
Para todos los grupos parlamentarios con representación continuada a lo largo del período histórico considerado (PSPV-PSOE, PPCV-PP y EUPV-IU), se constata la pauta en función de la cual se certifica la mayor tasa de circulación entre las diputadas que entre los diputados, siendo las diferencias, de mayor a menor gap comparativo por sexos y según los distintos grupos parlamentarios, según sigue:

$1^{\circ}$. PSPV: 0.23 .

$2^{\circ}$. PPCV: 0.01.

$3^{\circ}$. EUPV: 0.17 .

Tabla 6. La tasa de circulación de las élites parlamentarias de las Cortes Valencianas, por grupos parlamentarios, sexos y legislaturas

\begin{tabular}{lcccccc} 
& \multicolumn{2}{c}{ PSPV } & \multicolumn{2}{c}{ PPCV } & \multicolumn{2}{c}{ EUPV } \\
& M & H & M & H & M & H \\
\hline II & 0.33 & 0.25 & 0.00 & 0.55 & - & 0.83 \\
III & 0.75 & 0.28 & 0.00 & 0.76 & Incorporación & 0.20 \\
IV & 0.60 & 0.35 & 0.93 & 0.64 & 0.75 & 0.17 \\
V & 0.87 & 0.81 & 0.54 & 0.34 & 0.50 & 0.67 \\
VI & 0.55 & 0.50 & 0.38 & 0.47 & 0.00 & 0.40 \\
VII & 0.41 & 0.36 & 0.45 & 0.59 & 1.00 & 1.00 \\
\hline Media ponderada & 0.63 & 0.46 & 0.60 & 0.59 & 0.86 & 0.69 \\
\hline
\end{tabular}

Fuente: Elaboración propia a partir de Archivo de las Cortes Valencianas.

\section{PSPV-PSOE}

Respecto de los sucesivos grupos parlamentarios del PSPV, las distintas etapas que ilustran las pautas diferenciadas por sexos muestra cómo la brecha o gap entre los índices de circulación para ambos sexos es la más elevada de entre el conjunto de los grupos parlamentarios y para toda la secuencia histórica, siendo del 0.63 para las diputadas y del 0.46 para los diputados (+ 0.17).

En primer lugar, cabe destacar que el índice de circulación experimenta tendencias evolucionan de forma conjunta o concordante en el tiempo para ambos grupos según sexo, aun con intensidades diferentes, produciéndose una tendencia contraria tan sólo en la IV Legislatura (1995-1999), en la que disminuyendo el índice de circulación entre las diputadas con respecto a la III Legislatura (1991-1995), se dará sin embargo un incremento del mismo entre los diputados.

El primer incremento significativo en la presencia de mujeres en la III Legislatura (19911995), pasando del $7.14 \%$ de diputadas en la anterior (II legislatura) al $20.00 \%$ en la III, vendrá a implicar un destacado, y por otra parte obvio, aumento del índice de circulación de una a otra legislatura, siendo del 0.75 entre las diputadas en contraste con el 0.28 de los diputados. El número de diputadas en el grupo parlamentario del PSPV-PSOE pasa de 3 a 9, mientras que el número de diputados disminuirá, si bien ligeramente, pasando de 39 en la II Legislatura (1987-1991) a 36 en la III (1991-1995). 
A partir de lo antedicho, en la V Legislatura (1999-2003) se evidenciará el más elevado índice de circulación de toda la secuencia histórica, tanto para diputados como para diputadas del grupo socialdemócrata, permitiendo ser interpretado tal comportamiento por los siguientes motivos:

- El mayor incremento en el número de diputadas, pasando del 28.13 \% en la IV Legislatura (1995-1999) al $42.86 \%$ en la V (1999-2003), lo cual implicará la presencia de un total de 15 diputadas respecto de las 9 de la legislatura previa con motivo de la adopción de las cuotas electorales de paridad voluntarias en el seno del PSPV-PSOE. Desde la IV Legislatura (1995-1999) el número de diputadas en los sucesivos grupos parlamentarios del PSPV fue incrementándose de forma ininterrumpida, pasando de las ya enumeradas 9 diputadas en dicha legislatura a las 15 (42.86 \%), 16 (47.06 \%) y 19 (50.00\%) de las V, VI y VII Legislaturas, respectivamente.

- El cambio de liderazgo operado entre Joan Lerma como Presidente de la Generalitat Valenciana (1982-1995) y su sucesor Antonio Asunción implicará una renovación del grupo parlamentario del PSPV-PSOE, particularmente entre los hombres que habían venido manteniendo su acta de diputado en el período de liderazgo de Joan Lerma y que, consecuentemente, habían registrado niveles de circulación en una media del 0.29 para las primeras tres legislaturas consideradas (II, III y IV, entre 1987 y 1995), como parte del período de liderazgo antedicho.

- Tal cambio de liderazgo coincidirá, además, con los sucesivos declives en los resultados electorales del PSPV-PSOE que vendrán a consolidarse a partir de la V Legislatura (19992003) tras haber perdido la mayoría absoluta en la IV Legislatura (1995-1999) y, de esta forma, materializarse su transcurso a la oposición.

- La pérdida del gobierno autonómico (Consell) por parte del PSPV-PSOE, junto con el correspondiente cambio de liderazgo en el seno de la organización según se ha descrito, variables directamente conectadas entre sí en el caso expuesto, serán los factores que, junto con la implementación de las cuotas voluntarias de paridad electoral y la incorporación de las mujeres del partido socialdemócrata a las Cortes Valencianas, contribuyen a interpretar el máximo índice de circulación para ambos sexos, pero, de forma especialmente significativa, entre las mujeres.

En la VI Legislatura (2003-2007), a pesar de que la tasa de circulación disminuirá a partir del cénit registrado en la previa (V Legislatura), se experimenta una tasa del 0.55 entre las diputadas y del 0.50 entre los diputados del PSPV. De esta forma, se revela la consolidación de la presencia de las mujeres desde un punto de vista relativo, mediando el cambio de liderazgo que nuevamente se producirá en la VI Legislatura entre Antonio Asunción y Joan I. Plà, en un contexto de permanente desgaste electoral a lo largo de las sucesivas convocatorias electorales que vendrán a sucederse a partir de la IV Legislatura (1995-1999), en la que el PSPV-PSOE obtendría un 34.3 \% de votos en comparación con el $43.3 \%$ de la legislatura anterior (III), perdiendo la mayoría absoluta en el seno de las Cortes Valencianas. 
En todo caso, de forma concomitante con los factores más arriba enumerados, cabe constatar el hecho de que la circulación entre las diputadas es, en cualquiera de los acontecimientos, y más allá de los distintos datos registrados, más elevada que entre los hombres, debido no sólo al incremento continuado en el número y porcentaje de las diputadas, sino sobre todo, a la renovación persistente de aquellas mujeres que resultan titulares del acta de diputada a lo largo de un menor número de legislaturas.

\section{EUPV-IU}

En relación a EUPV-IU es preciso comenzar destacando el impacto metodológico que se deriva de la menor cantidad de casos y la proyección que de dicha circunstancia se deriva a la hora de evaluar la influencia que sobre la tasa de circulación tienen los cambios de liderazgo, la renovación, la incorporación, el acceso efectivo y la oportunidad, ésta última en función de los resultados electorales y del número de escaños obtenidos. De todo ello se deriva una variabilidad destacada de los datos por cuanto se refiere a las sucesivas tasas de circulación que se registran en las correspondientes legislaturas y, por tanto, en los sucesivos grupos parlamentarios de EUPV-IU, según se expone a continuación.

Los dos principales factores que permiten explicar las variaciones estadísticas en relación con la tasa de circulación para cada una de las legislaturas viene dado por los factores de oportunidad (los resultados electorales obtenidos) y, en este sentido, por el índice de acceso efectivo al cargo, así como por otra parte, debido a los cambios de liderazgo en el seno de la organización y, como consecuencia, en el propio grupo parlamentario.

En primer lugar, cabe considerar el hecho de que, a diferencia de los datos de que se tiene constancia respecto de los diputados de EUPV-IU, y que se remontan al principio de la secuencia histórica (II Legislatura), la tasa de circulación de las mujeres tan sólo se puede analizar a partir de la IV Legislatura (1995-1999), tras la incorporación de las diputadas por EUPV-IU a las Cortes Valencianas en la legislatura previa, siendo por tanto en EUPV-IU donde la incorporación de las mujeres se da de forma más tardía en comparación con el conjunto restante de los grupos parlamentarios considerados (PSPV-PSOE y PPCV-PP).

La tasa de circulación en EUPV-IU ha venido claramente influenciada pues, por los resultados electorales obtenidos y, de forma particular, a la luz de los escaños ostentados por mujeres. Así, el segundo dato más elevado de la tasa de circulación entre las mujeres se producirá, por detrás del de la renovación absoluta (tasa de circulación 1.00) de la VII Legislatura, en la IV (0.75), debido a la incorporación de tres nuevas diputadas y la continuidad de una de ellas con respecto a la legislatura previa (1995-1999).

La tasa de circulación llegará a ser de 0.00 en la VI Legislatura (2003-2007), en la que no se produjo la incorporación de ninguna nueva diputada, pero sí la continuidad de la única que en dicha legislatura ostentará un acta de diputada procedente de la IV Legislatura (1995-1999). Será finalmente en la última de las legislaturas consideradas en la secuencia histórica (2007-2011), cuando la tasa de circulación será de 1.00, lo cual implica la renovación absoluta del grupo parlamentario de EUPV-IU respecto de la legislatura previa, con la 
incorporación de 4 diputadas y de 3 diputados, constituyendo de esta forma la presencia de las mujeres el $57.14 \%$.

En este último caso (VII Legislatura) concurren los factores más abajo detallados, conforme a los cuales interpretar el comportamiento de la tasa de circulación; a saber:

- El segundo mejor resultado electoral de la formación (195.116 votos y 7 escaños) tras los obtenidos en la IV Legislatura (273.030 votos y 10 escaños), lo cual permite un mayor impacto del factor de oportunidad y con ello de un mayor acceso, tanto de hombres como de mujeres, al correspondiente grupo parlamentario, generándose así una significativa tasa de circulación debido a la renovación y al evidenciado acceso; y,

- El cambio de liderazgo de Joan Ribó (V y VI Legislaturas) al de Gloria Marcos (VII Legislatura) encabezando ésta última la candidatura de Valencia en cuanto que candidata a la Presidencia de la Generalitat y Coordinadora General de la coalición EUPV, una de las formaciones inicialmente integrantes de la coalición política COMPROMÍs.

Entre los diputados de EUPV, las tasas de circulación han mostrado tres vértices:

- En primer lugar, el derivado de la propia renovación de 5 de los 6 diputados que habían ocupado el acta parlamentaria en la I Legislatura (1983-1987) y que habían constituido un grupo parlamentario exclusivamente masculino;

- En segundo lugar, debido al cambio de liderazgo que operará en la V Legislatura (19992003) con la incorporación de Joan Ribó y la sustitución de Albert Taberner, a pesar del decreciente resultado electoral obtenido (de 6 a 3 diputados y de 4 a 2 diputadas); y,

- Finalmente, la renovación absoluta y la tasa de circulación de 1.00 entre los diputados, del mismo modo que se da con respecto a las diputadas, con motivo no sólo del cambio de liderazgo, sino también del mejor y más sólido resultado electoral en relación a los comicios previos.

En cualquier caso, para el conjunto de la secuencia histórica se produce una tasa de circulación más elevada entre las mujeres que entre los hombres (con una diferencia de 0.17 puntos), registrándose así la segunda mayor brecha por sexos de entre el conjunto de los grupos parlamentarios estudiados tras el ya analizado del PSPV (0.23).

Se puede concluir, según lo expuesto, que la tasa de circulación entre las diputadas es más elevada debido a la incorporación de las mujeres a la actividad parlamentaria en los sucesivos grupos parlamentarios de EUPV, y como consecuencia del destacado incremento en cuanto al resultado electoral que acompaña aquél acontecimiento de incorporación de las mujeres en la IV Legislatura (1995-1999) y el reiterado resultado electoral obtenido en la VII Legislatura (2007-2011). Asimismo, cabe señalar que el factor de oportunidad también resulta relevante y significativo a la hora de explicar la tasa de circulación entre las diputadas de los grupos parlamentarios de EUPV-IU, siendo menos destacado el peso que dicha variable ha tenido en comparación con el impacto de los cambios de liderazgo, factor que se puede reseñar de forma destacable sin embargo en la VII Legislatura (2007-2011). 


\section{PPCV-PP}

En relación al comportamiento de las cifras ligadas a la tasa de circulación en el seno de los sucesivos grupos parlamentarios del PPCV-PP, se experimenta la mínima de las diferencias que entre sexos se produce para cada grupo parlamentario. Ello se debió, fundamentalmente, a la elevada circulación que se dará de forma indistinta entre diputados y diputadas como consecuencia de la alta renovación registrada en las primeras legislaturas (II, III y IV), en las que hasta la victoria con mayoría absoluta del PPCV-PP en la IV Legislatura, se vendrían sucediendo constantes procesos de restructuración y sustitución entre las diferentes organizaciones partidistas ubicadas en el espectro ideológico del partido, tanto en el entorno político del mismo, como en el propio seno del PPCV-PP. Todo ello cabe ser interpretado como resultado de la tardía consolidación del PPCV-PP hasta la absorción electoral de las fuerzas de centro derecha (CDS) y conservadoras de identidad regionalista (UV) en la V Legislatura (1999-2003), así como la definitiva transformación de AP en el contemporáneo PPCV-PP.

A partir del instante descrito en el párrafo anterior y hasta la VII Legislatura (2007-2011), las cifras de la tasa de circulación serán de las más bajas de toda la secuencia histórica, coincidiendo de forma consecutiva y sostenida con las de la V y VI Legislaturas. No obstante lo antedicho, en la VI Legislatura se producirá una tasa de circulación más elevada con respecto a la previa en coincidencia con el cambio de liderazgo entre Eduardo Zaplana y Francisco Camps.

Los diferentes índices estadísticos asociados a la tasa de circulación de las mujeres en el seno del PPCV-PP vienen asociados a la incorporación de las mismas a las Cortes Valencianas en la IV Legislatura (1995-1999), pasando de tan sólo una (en la III Legislatura) a ocho en la IV. Dicha circunstancia se dará en coincidencia con el cambio de liderazgo acontecido en dicha convocatoria electoral (de Pedro Agramunt a Eduardo Zaplana), así como con el incremento histórico en el resultado electoral por parte del PPCV-PP, que pasará de los 558.617 votos y los 31 escaños en la III Legislatura (1991-1995), a 1.013.859 votos y 42 escaños en la IV Legislatura (1995-1999), protagonizando el cambio en la mayoría parlamentaria en el seno de las Cortes Valencianas y dando apoyo y constitución al Gobierno Autonómico valenciano (Consell).

Dicho factor de oportunidad en cuanto a la incorporación de las mujeres se manifiesta de nuevo de una forma especialmente significativa a la hora de interpretar, no sólo la mayor presencia de las mujeres en las instituciones representativas, sino también y precisamente debido a este factor, la mayor circulación, que será de 0.54 en la V Legislatura (1999-2003) en coincidencia y coherencia con la incorporación de 14 diputadas en dicho grupo parlamentario, consolidándose así la presencia de las mujeres en el mismo por encima del umbral de masa crítica, pasando del $19.05 \%$ en la legislatura previa, al $38.76 \%$ en la V.

Finalmente, la VII Legislatura (2007-2011) vendrá a coincidir con un incremento significativo en la tasa de circulación respecto de la legislatura previa, tanto entre los diputados como entre las diputadas, consecuencia ello de la consolidación del liderazgo de Francisco Camps 
frente al previo de Eduardo Zaplana, manifestándose tal circunstancia mediante la renovación que tras una legislatura de gobierno de Francisco Camps se dará de forma efectiva en la convocatoria electoral del año 2007 con la renovación de todos aquellos diputados de la VI Legislatura (2003-2007) que no repitieron como candidatos en la VII (2007-2011) ante la incorporación de nuevos/as candidatos/as y cargos ligados al liderazgo de Francisco Camps.

En el caso del PPCV-PP, los factores que contribuyen a explicar la elevada tasa de circulación de las mujeres vienen dados por el impacto que se deriva de una más tardía incorporación de las mujeres en general a la actividad parlamentaria, así como al hecho de que dicha incorporación se produce en un momento de oportunidad caracterizado por las sucesivas mayorías absolutas del PPCV-PP. Las tasas de elevada circulación entre los diputados no se diferencian de las diputadas en igual medida e importancia con que acontece en el PSPV-PSOE y en EUPV-IU. Ello es debido a la variabilidad del liderazgo en los precedentes momentos de peores resultados electorales acumulados, a los procesos de reorganización partidista y orgánica en el espectro ideológico, así como a la confluencia de numerosos cambios de liderazgo (de Agramunt a Zaplana en primer lugar; y de Zaplana a Camps en segundo término) momentos que vendrán a coincidir además con la mayor incorporación de diputadas a las Cortes Valencianas con motivo de las sucesivas mayorías absolutas que implicarán un ascenso continuado en el período abarcado entre las IV, V, VI y VII Legislaturas.

\subsection{Los factores explicativos de la circulación parlamentaria por sexos y grupos par- lamentarios (1983-2011)}

Según todo lo ya expuesto, cabe enumerar los siguientes factores en tanto que incidentes en la tasa de circulación del conjunto de los diputados y diputadas de las Cortes Valencianas:

- El cambio de liderazgo en el seno de la organización política y, de esta forma, en el correspondiente grupo parlamentario o gobierno (en este último caso cuando confluya una mayoría relativa o absoluta en las Cortes Valencianas que dé sustento al correspondiente gobierno autonómico); y,

- Los factores de oportunidad o de mejores resultados electorales que además, en el caso de las mujeres se asociarán con una mayor presencia de las mismas y de esta forma, no tanto como consecuencia de la renovación respecto del recambio en las élites de la legislatura anterior, sino debido a la incorporación de un mayor número de mujeres al correspondiente grupo parlamentario.

No obstante, el impacto de cada uno de estos factores varía de un grupo parlamentario a otro, y del mismo modo también entre sexos. De esta forma, se constata que los cambios de liderazgo resultan más significativos a la hora de explicar la mayor tasa de circulación entre los diputados, mientras que la relativa a las diputadas viene dada fundamentalmente, por la variación ascendente en el resultado electoral obtenido y el factor de oportunidad que ello genera y evidencia en la incorporación de un mayor número y porcentaje de mujeres en tanto que diputadas. 
En relación al PSPV-PSOE y a EUPV-IU, los factores comunes que permiten explicar la mayor tasa de circulación entre las mujeres vienen dados por el hecho de la incorporación de las mismas con motivo de las cuotas voluntarias introducidas por las formaciones. En el caso de EUPV-IU, junto con dicha adopción de las cuotas voluntarias cabe identificar los indicadores de oportunidad, es decir, el más favorable electoral obtenido en las sucesivas convocatorias, permitiendo el acceso de más diputadas a los grupos parlamentarios propios de la coalición. De igual forma, los cambios de liderazgo resultan más significativos entre los diputados que no entre las diputadas, de tal suerte que las nivelación entre las tasas de circulación para cada uno de los sexos, en casos de cambio de liderazgo, se debió más bien a la confluencia, en esos casos, de una mayor incorporación de la mujer como consecuencia del mayor acceso efectivo.

En el caso del PPCV-PP cabe destacar la importancia, no sólo del factor oportunidad que va a permitir a las mujeres el acceso paulatino a las Cortes Valencianas al albur de los resultados electorales obtenidos en sucesivas convocatorias electorales, y que se dará en el momento más significativo que supondrá la mayoría absoluta cosechada por el PPCV-PP en la IV Legislatura y la superación del umbral de masa crítica en cuanto a la presencia de las mujeres se refiere. Junto con dicho factor de oportunidad, el hecho de que la diferencia entre las tasas de circulación de diputados y diputadas sea la más baja de entre los tres grupos parlamentarios considerados, cabría ser interpretada por el hecho de que la tasa de circulación de las mujeres es la más baja en relación al PSPV-PSOE y a EUPV-IU. Dicha circunstancia coincide con la elevada tasa de circulación entre los diputados fruto del impacto ocasionado por la lenta consolidación del liderazgo dentro de la formación y la fragmentación de las fuerzas de centro derecha en torno al PPCV-PP en las primeras legislaturas.

Por ello, cabe advertir como principal conclusión del presente epígrafe que cuando la aproximación a la tasa de circulación de las élites políticas se realice desde la perspectiva de género, debiérase considerar la concurrencia de factores explicativos tales como:

- La fragmentación de las fuerzas políticas en el marco de un espectro ideológico común, así como en los sucesivos procesos de convergencia y de restructuración, crisis o estabilidad institucional y orgánica;

- La oportunidad, y por tanto, la incidencia del resultado electoral que de forma creciente, estable o menguante vayan obteniendo las distintas organizaciones políticas;

- La consolidación o variabilidad en las pautas del liderazgo de la correspondiente organización partidista y en consecuencia, en el ámbito institucional-parlamentario; y,

- Como resultado de los dos últimos factores señalados (oportunidad y cambio de liderazgo) en conjunción, el hecho de que la formación política correspondiente pase a ocupar posiciones de gobierno o de oposición en distintos sentidos. 


\subsection{Estudio comparativo cross-time (1983-2011) por sexos y grupos parlamentarios de las pautas de permanencia de las élites parlamentarias de la Cortes Valencianas}

Conforme a las tablas 9, 10 y 11, la permanencia en el cargo resulta superior entre los diputados varones en contraposición con las tendencias operadas sobre las diputadas a lo largo de toda la secuencia histórica (1983-2011) y para el conjunto de los grupos parlamentarios. De esta forma, a la luz de los datos expuestos más abajo, en la medida en que se avanza en la acumulación de legislaturas en las que un diputado o cargo público permanece en el cargo de forma continuada, ininterrumpida y correlativa, la brecha o diferencia entre las pautas de permanencia según sexos se acrecienta y amplifica.

A excepción de la tasa de permanencia ligada a la titularidad del acta parlamentaria a lo largo de dos legislaturas consecutivas, en que la diferencia por sexos es la mínima, en el resto de los casos (mayor número de legislaturas acumuladas ininterrumpidamente), la diferencia por sexos manifiesta una tendencia creciente y divergente entre sí. De este modo, a mayor número de legislaturas en el cargo, más elevada es también la diferencia en función del sexo, siendo menor entre las diputadas hasta el punto de que para una permanencia de seis legislaturas consecutivas, tan sólo se obtienen datos de diputados (hombres), sin que ninguna diputada haya ostentado el acta parlamentaria a lo largo de tal período de forma ininterrumpida.

Considerando los datos acumulados para el conjunto de la secuencia histórica, el porcentaje de diputadas que han ejercido el cargo durante una única legislatura es superior al de los diputados en el caso de EUPV-IU (77.78 \% frente al $61.11 \%$ ), al igual que sucede en el PSPV-PSOE (52.17 \% frente al $47.83 \%$ ), siendo algo menor la duración de los diputados con respecto a las diputadas en el caso del PPCV-PP (55.73\% diputados vs. $51.85 \%$ diputadas), como consecuencia en este último caso de la mayor equivalencia entre las tasas de circulación de diputados y diputadas en los sucesivos grupos parlamentarios del PPCV-PP, según se ha expuesto más arriba.

En el PSPV-PSOE es menor la permanencia en el cargo por parte de las mujeres, de ahí que el ejercicio del cargo en la secuencia ininterrumpida en el seno de la horquilla comprendida entre una y tres legislaturas sea superior entre las diputadas, siendo equivalente la permanencia entre diputados y diputadas cuando se trata de, igualmente, tres legislaturas de permanencia. Será a partir de la acumulación superior a las cuatro legislaturas en el supuesto en que se pondrá de manifiesto la mayor permanencia de los diputados con cifras del 8.69 \% en las cuatro legislaturas, del $1.74 \%$ en las cinco legislaturas y del $0.87 \%$ en el umbral de las seis legislaturas. Tan sólo en la permanencia de cinco legislaturas aparece un caso entre las diputadas (el $2.17 \%$ ), frente a dos casos masculinos con un $1.74 \%$ de los mismos en dicho nivel de permanencia.

Del mismo modo, los sucesivos grupos parlamentarios de EUPV-IU representan una tendencia en virtud de la cual el 77.78 \% de los casos de entre sus diputadas se ubican en la ostentación del acta parlamentaria durante una única legislatura, registrándose el resto (dos casos, el 22.22 \%) en el umbral de las tres legislaturas. Si bien, entre los diputados se obtiene 
una mayor dispersión estadística, de tal suerte que a pesar de que tan sólo un caso llega al nivel de las cinco legislaturas ininterrumpidas (el $5.56 \%$ ), el restante $94.44 \%$ se reparte en los niveles de una (61.11\%), dos (1.11\%) y tres legislaturas (22.22 \%).

En un sentido similar cabe interpretar los resultados obtenidos en cuanto a la permanencia entre los diputados de UV, con registros de permanencia de una (69.22 \%), dos (15.39 \%) y tres legislaturas (15.39 \%). El único caso de la diputada de UV ostentará el cargo a lo largo de las tres legislaturas en las que la formación tendrá representación parlamentaria (el 100.00 $\%$, por tanto).

Finalmente, en el PPCV-PP, debido a los factores de la más arriba testimoniada elevada circulación de los diputados, la permanencia en tan sólo una legislatura es mayor entre los diputados (55.73 \%) con respecto y en comparación a las diputadas (51.85\%). Conforme a la misma tendencia sucede entre diputados y diputadas, aunque con una mayor diferencia porcentual de 10.11 puntos, por cuanto a la permanencia durante dos legislaturas se refiere (21.37 \% y 31.48 \%, respectivamente). La permanencia de los diputados del grupo parlamentario popular será superior, no obstante, al de las diputadas, en el nivel de las tres (9.26 $\%$ en diputadas frente al $16.79 \%$ en los diputados) y cinco legislaturas (en este último caso con registros únicamente dados entre los hombres, con un índice del 1.53 \%).

Tabla 7. Media ponderada de la tasa de circulación de las élites parlamentarias de las Cortes Valencianas, por sexos y legislaturas, para el conjunto de los grupos parlamentarios

\begin{tabular}{lllc} 
& M & H & Diferencia \\
II & 0,33 & 0,48 & $-0,15$ \\
\hline III & 0,75 & 0,59 & 0,16 \\
\hline IV & 0,82 & 0,56 & 0,26 \\
\hline V & 0,69 & 0,61 & 0,08 \\
\hline VI & 0,47 & 0,48 & 0,01 \\
\hline VII & 0,54 & 0,57 & 0,03 \\
\hline TOTAL & 0,64 & 0,55 & 0,09 \\
\hline
\end{tabular}

Fuente: Elaboración propia a partir de Archivo de las Cortes Valencianas.

Tabla 8. Tasa de circulación de las élites parlamentarias de las Cortes Valencianas, por grupos parlamentarios, sexos y legislaturas

\begin{tabular}{lccccccc} 
& & PSPV & & & PPCV & \multicolumn{2}{c}{ EUPV } \\
\cline { 2 - 7 } II & M & H & M & H & M & $H$ \\
III & 0,33 & 0,25 & 0,00 & 0,55 & - & 0,83 \\
\hline IV & 0,75 & 0,28 & 0,00 & 0,76 & Incorporación & 0,20 \\
V & 0,60 & 0,35 & 0,93 & 0,64 & 0,75 & 0,17 \\
VI & 0,87 & 0,81 & 0,54 & 0,34 & 0,50 & 0,67 \\
VII & 0,55 & 0,50 & 0,38 & 0,47 & 0,00 & 0,40 \\
Media ponderada & 0,41 & 0,36 & 0,45 & 0,59 & 1,00 & 1,00 \\
\hline
\end{tabular}

Fuente: Elaboración propia a partir de Archivo de las Cortes Valencianas. 
Circulación y permanencia de las élites... | Bernabé Aldeguer Cerdá

Tabla 9. Tasa de circulación de las élites parlamentarias de las Cortes Valencianas, por grupos parlamentarios, sexos y legislaturas

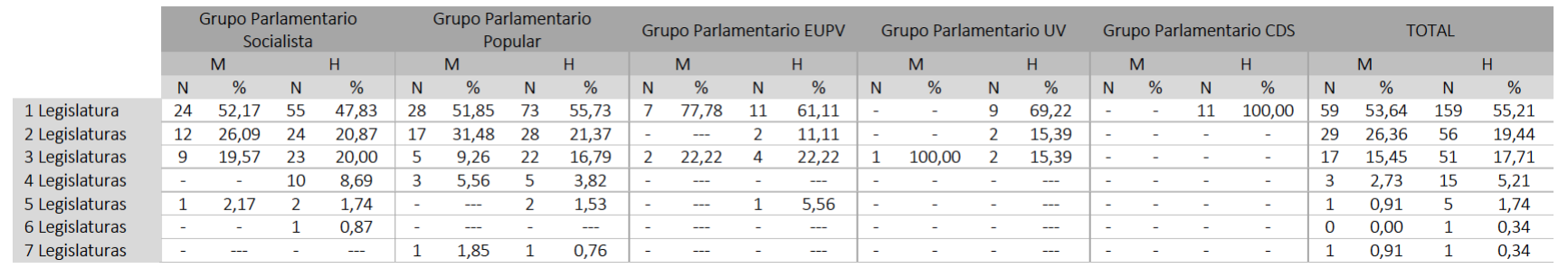

Fuente: Elaboración propia a partir de Archivo de las Cortes Valencianas.

Tabla 10. La permanencia de las élites parlamentarias autonómicas valencianas. Las diputadas (1983-2011)

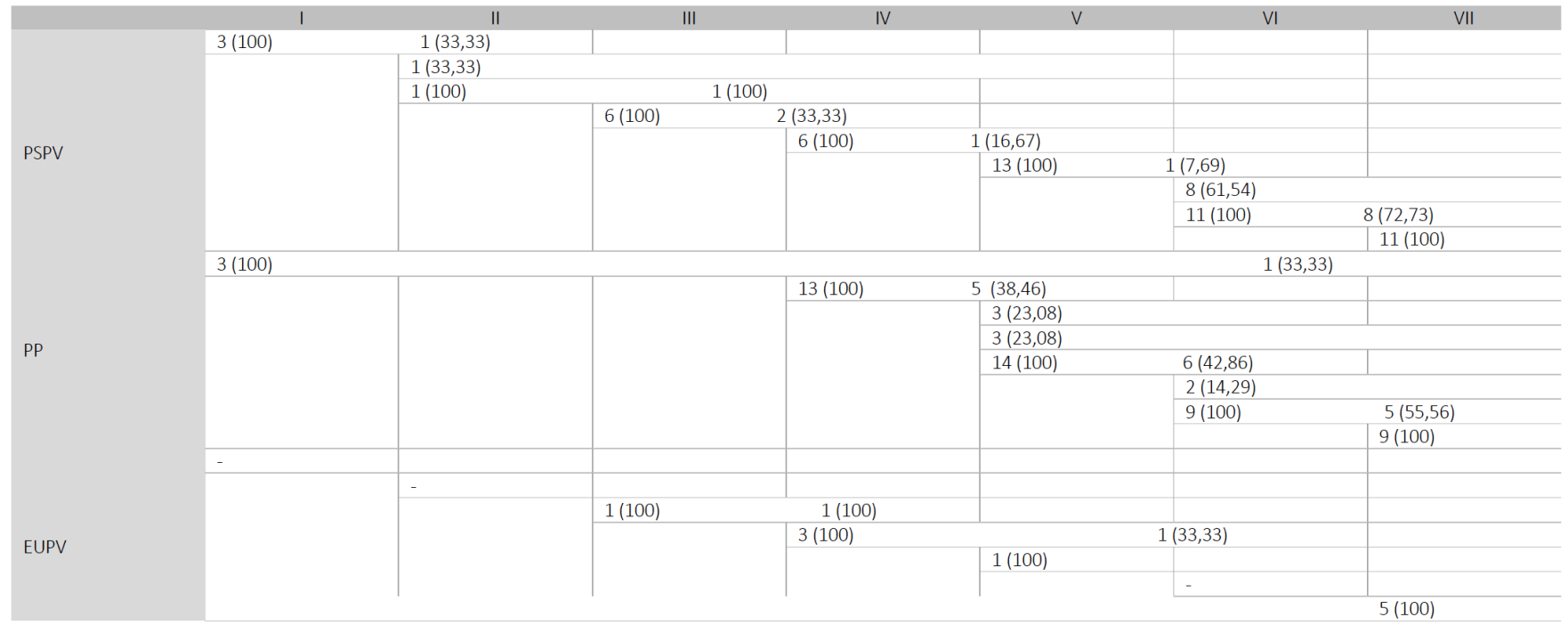

Fuente: Elaboración propia a partir de Archivo de las Cortes Valencianas.

UV tendrá una diputada que accederá por primera vez en la II Legislatura y que permanecerá como diputada a lo largo de las dos siguientes Legislaturas, de tal forma que son tres las Legislaturas que le caben imputar; a saber: I (1983 - 1987), II (1987 - 1991) y III (1991 - 1995). Aparecen más diputadas que en las tablas donde se hace constar el número de diputadas por Grupo Parlamentario, ya que en este caso se ha computado, por motivo de la presentación de los datos estadísticamente recopilados, el conjunto de las diputadas, también las que se incorporan a lo largo de la Legislatura. 
Tabla 11. Permanencia de las élites parlamentarias autonómicas valencianas. Los diputados (1983-2011)

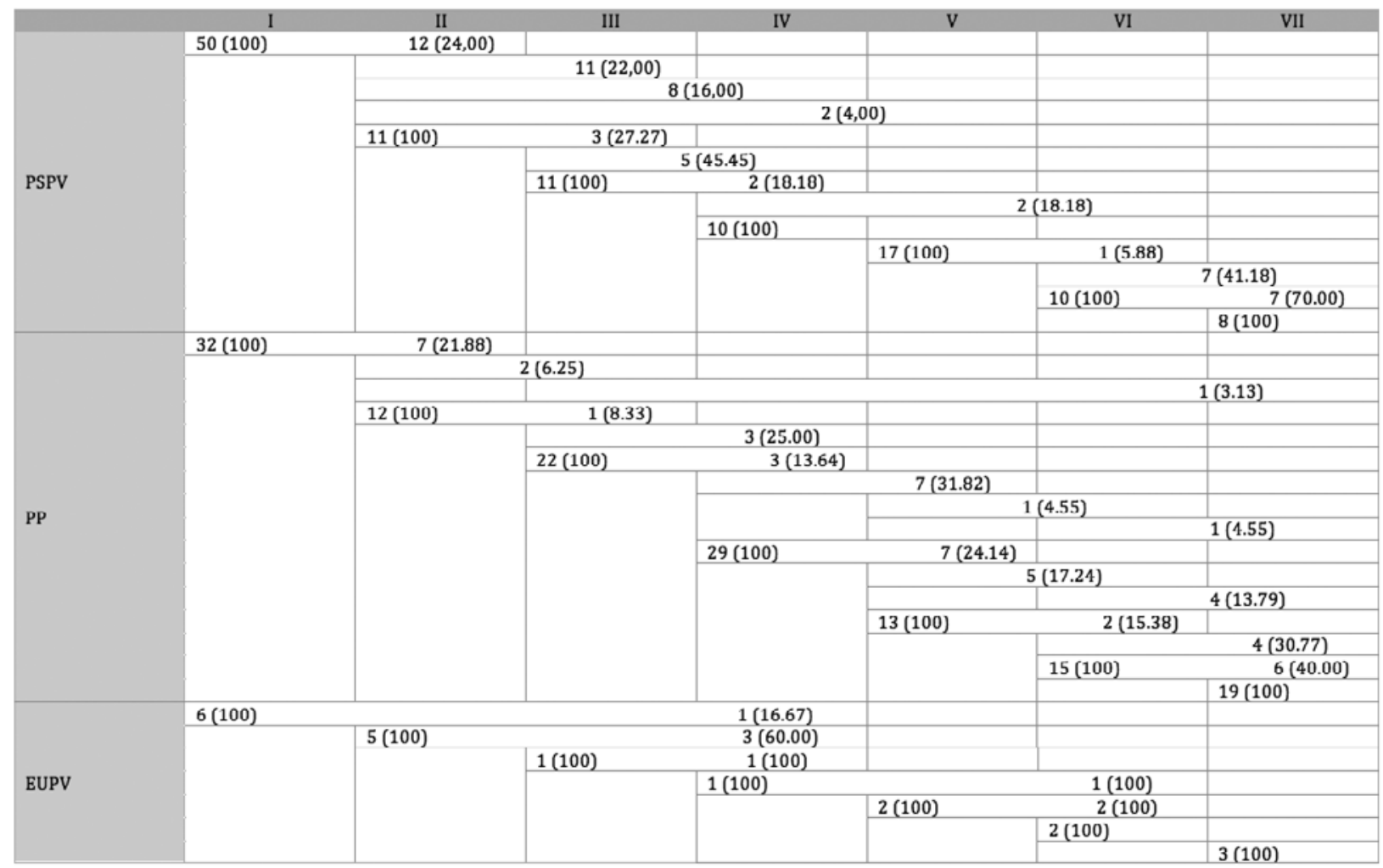

CDS tendrá representación únicamente en la II Legislatura. Por su parte, UV estará presente en las Cortes Valencianas a lo largo de tres legislaturas, de forma que las pautas de continuidad y discontinuidad son las siguientes:

\begin{tabular}{|c|c|c|}
\hline II & III & IV \\
\hline \multirow[t]{4}{*}{$6(100)$} & $1(16,67)$ & \\
\hline & \multicolumn{2}{|c|}{$2(33,33)$} \\
\hline & $4(100)$ & $1(25,00)$ \\
\hline & & $2(100)$ \\
\hline
\end{tabular}

Fuente: Elaboración propia a partir de Archivo de las Cortes Valencianas.

\section{CONCLUSIONES}

En el marco de la significativa relevancia asociada al estudio de las pautas de circulación y permanencia en el cargo como parte sustancial de las investigaciones sobre las élites políticas, la aproximación a tal objeto de estudio desde la perspectiva de género cobra particular importancia teniendo en cuenta las diferencias comparativas que eventualmente divergen el ejercicio del cargo por parte de mujeres y hombres.

En este sentido, el presente trabajo partía del propósito destinado a analizar las eventuales diferencias que entre mujeres y hombres pudiera darse, en tanto que cargos públicos, desde el análisis de la circulación y la permanencia, identificando los factores que permitan explorar el comportamiento de la citada variable. Partiendo de la hipótesis acerca de la mayor tasa de circulación entre las mujeres con respecto a los hombres, los cuales permanecerían en el cargo de forma más estable a lo largo del tiempo, a la luz de investigaciones previas, el trabajo ha permitido identificar algunos factores que podrían ayudar a analizar este objeto de estudio; a saber: la fragmentación de las fuerzas políticas en el marco de un espectro 
ideológico común, así como en los sucesivos procesos de convergencia y de restructuración, crisis o estabilidad institucional y orgánica; la oportunidad, y por tanto, la incidencia del resultado electoral que de forma creciente, estable o menguante vayan obteniendo las distintas organizaciones políticas; la consolidación o variabilidad en las pautas del liderazgo de la correspondiente organización partidista, y en consecuencia, en el ámbito institucional-parlamentario; y, como resultado de los dos últimos factores señalados (oportunidad y cambio de liderazgo) en conjunción, el hecho de que la formación política correspondiente pase a ocupar posiciones de gobierno o de oposición en distintos sentidos.

La investigación, partiendo de un estudio de caso a nivel autonómico (Cortes Valencianas) a lo largo de una secuencia cronológica que comprende y abarca un total de siete legislaturas, analiza el desigual ejercicio del cargo por parte de mujeres y hombres, aun habiendo, desde el punto de vista cuantitativo y descriptivo, adquirido unos niveles equilibrados de participación política. Se incide, de esta forma, en la idoneidad de proyectar trabajos similares que, incluso desde una perspectiva multinivel y según partidos políticos, permitan arrojar luz sobre los resultados acopiados de forma paulatina en los últimos años tras la consolidación de la presencia de mujeres en las instituciones públicas, mejorando en su caso las metodologías a implementar y, en consecuencia, la contribución a la actualización conceptual sobre la cuestión.

\section{BIBLIOGRAFÍA}

Blondel, J., Müller-Rommel, F. \& Malová, D. (2007). Governing New European Democracies. Basingstoke: Palgrave McMillan.

Coller, X. (1999). “Circulación y conflicto en la élite política. El caso valenciano”, Revista Valenciana d’Estudis Autonòmics, núm. 29, pp. 193-221.

Comisión Europea (2009). Women in European politics - time for action. Bruselas: European Communities.

Connell, R. W. (1994). “The State, Gender and Sexual Politics”, en Radtke, H. L. \& Henderikus, J. S. Power / Gender. Social Relations in Theory and Practice. London: Sage.

Dahlerup, D. \& Freidenvall, L. (2008). Electoral Gender quotes systems and their implementation in Europe. Brussels: European Parliament.

Githens, M. (2003). “Accounting for Women’s Political Involvement: The Perennial Problem of Recruitment”, en Carroll, S. J. Women and American Politics: New questions, new directions. Oxford: Oxford University Press, pp.: 33-52.

Lois González, M. (2007). "Mujeres y toma de decisiones. Una aproximación a la literatura especializada”, en Diz Otero, I. \& Lois González, M. (eds.), Mujeres, instituciones y política. Barcelona: Bellaterra. 
Lovenduski, J. (2005). Feminizing politics. Cambridge: Polity Press.

Mansbridge, J. J. (1998), “Women, government and the common good”, en Phillips, A., Feminism and politics, New York: Oxford University Press, pp. 142-150.

Morán, $M^{a}$. L. (1989). "Un intento de análisis de la clase parlamentaria española: elementos de renovación y permanencia (1977 - 1986)”, en Revista Española de Investigaciones Sociológicas, 45, pp. 61-84.

Naciones Unidas (1992). Declaración de Atenas. Adoptada en la primera Cumbre Europea “Mujeres en el Poder", celebrada en Atenas el 3 de noviembre de 1992.

Naciones Unidas (2005). Declaración de Beijing y Plataforma para la Acción: Beijing +10. Madrid: Ministerio de Asuntos Sociales.

Ortega, C., Torres, J. y Trujillo, J. M. (2011). "La igualdad efectiva entre mujeres y hombres. Un estudio de las elecciones al Congreso de los Diputados de 2008”, en Psicología Política, 42, pp: 117-139.

Pareto, W. (1932). Traité de Sociologie. París: Payot.

Pareto, W. (1980). Forma y equilibrio sociales. Alianza Editorial: Madrid.

Pastor, R. (2011). Género, élites políticas y representación parlamentaria en España. Valencia: Tirant lo Blanch.

Paxton, P. \& Hughes, M. (2007). Women, Politics and Power. A Global Perspective. London: Sage.

Phillips, A. (1998). Feminism and politics. New York: Oxford University Press.

Pitkin, H. F. (1985). El concepto de representación. Madrid: Centro de Estudios Constitucionales.

Putnam, R. E. (1976). The comparative Study of Political Elites. New Jersey: Prentice-Hall.

Rodríguez Teruel, J. (2011). Los ministros de la España Democrática. Madrid: CEPC.

Sapiro, V. (1998). "Feminist Studies and Political Science-and Viceversa", en Phillips, A. Feminism \& Politics. Oxford: Oxford University Press.

Sevilla Merino, J. (1997). "Las presencia de las mujeres en los Parlamentos: las Cortes Valencianas”, en Corts: Anuario de Derecho Parlamentario, nº . 4, pp. 369-411.

Sevilla Merino, J. (2004). Mujeres y ciudadanía: la democracia paritaria. Valencia: Universitat de València - Institut Universitari d’Estudis de la Dona. 
Squires, J. (2007). The New Politics of gender equality. Palgrave: Macmillan.

Uriarte, E. (1997). "El análisis de las élites políticas en las democracias”, en Revista de Estudios Políticos (Nueva Época), núm. 97, pp.: 249 - 275.

Verge, T. (2006). "Mujeres y partidos políticos en España: las estrategias de los partidos políticos y su impacto institucional, 1978-2004”, en Revista Española de Investigaciones Sociológicas, núm. 115, pp. 165-196.

Verge, T. (2009). Dones a les institucions polítiques catalanes: el llarg camí cap a la igualtat (1977 - 2008). Barcelona: ICPS-UAB. 